\title{
Double-Cavity Fabry-Perot Resonators Based on One-dimensional Silicon Photonic Crystals
}

\author{
Vladimir Tolmachev, ${ }^{1}$ Anna Baldycheva ${ }^{2}$ and Tatiana S. Perova ${ }^{3,4, a)}$ \\ ${ }^{I}$ Department of Solid State Electronics, Ioffe Institute, Polytechnicheskaya 26, St.-Petersburg 194021, Russia \\ ${ }^{2}$ University of Exeter, College of Engineering Mathematics and Physical Sciences, Exeter, EX4 4QF, UK \\ ${ }^{3}$ School of Engineering, Trinity College Dublin, The University of Dublin, Dublin 2, Ireland \\ ${ }^{4}$ ITMO University, St Petersburg, 197101, Russia \\ ${ }^{\text {a)}}$ Corresponding author: perovat@tcd.ie
}

\begin{abstract}
In this work we report on optical properties of Fabry-Pérot (FP) resonator based on Si-air one-dimensional photonic crystal (1D PC) with coupled double-cavity modes (or defects). These defects obtained by infiltration of the air-cavities of refractive index $\left(n_{\text {Air }}\right)$ with the filler of tunable refractive index. In the periodic structure of $1 \mathrm{D}$ PC, the filling of two defined grooves with a filler with a refractive index different from $n_{\text {Air }}$ leads to the appearance of two resonant modes, the position of which can be adjusted purposefully with changing of $n$ filler. In comparison with $(\lambda / 2)$ air resonators, the splitting of the doublet increases, which is explained by the increase in the coupling between the resonant modes due to the decrease in reflection $R$ of the internal mirror. The coupled FP resonators design is CMOS compatible and has potential for application in tuning of individual transmission bands in wave-division multiplexing systems as well as for multiple narrow filters in the wide infrared spectral range.
\end{abstract}

\section{INTRODUCTION}

For effective separation of certain frequencies, multimode filters are used that can be obtained by creating a cavity of extended size in a Fabry-Pérot (FP) resonator, as well as using cascaded and coupled resonators. Cascade structure consist of separate components (FP filters) connected in series, while changing the optical properties of each of them does not affect the properties of the neighboring filters [1-5]. The development of integrated compact multi-channel optical filters for various applications is a rapidly developing area. Coupled microresonators are used in waveguide structures [6], in ring resonators [7], which can be tuned by injecting carriers with $\Delta n<10^{-4}$, so they have a relatively small tunable range. Thanks to the use of liquid crystals with a large range of $\Delta n$, the tuning of the resonance modes has been significantly extended, for example, in the case of the cascade resonator [2]. In Ref. [8] the calculation of the optical equalizer (tunable filter) is proposed with the possibility of changing the shape of several interference bands by changing $n$ in different periods of the one-dimensional photonic crystal (1D PhC) structure. The optical properties of the system of two coupled Fabry-Pérot microresonators were investigated in Ref. [9], where the interaction of localized photon states was considered, leading to a splitting of the optical modes of the system. In this work, the coupling parameter (the reflection $R$ value of the common mirror) was revealed, which characterizes the interaction force between the modes. The decrease in $R$ led to an increase in the splitting of the peaks in the spectrum. Using the theoretical prerequisites for the creation of coupled FP resonators and assuming the possibility of individual tuning of $n$ in each resonator, a 1D PhC structure with two defects and different options of controlling the position of the doublet of resonance modes were investigated in this work

\section{RESULTS AND DISCUSSIONS}




\section{Calculations of Double Coupled Fabry-Pérot resonators}

The introduction into 1D PhC instead of single cavity (as shown in Fig. 1a) two cavities separated by the common mirror, leads to the formation of double-cavity conjugated FP resonators (Fig. 1b). Transfer Matrix Method (TMM) was used for simulations of the Si-Air and Si-Liquid Crystal (LC) 1D PhC structures with two defects. The transfer matrix for double-cavity resonators (S) for calculations of the transmission spectrum, $T$, can be written as

$$
\mathrm{S}=\mathrm{H}(\mathrm{LH})^{\mathrm{m}} \mathrm{L}_{\mathrm{res}}\left[(\mathrm{HL})^{\mathrm{mo}} \mathrm{H}\right] \mathrm{L}_{\mathrm{res} 2}(\mathrm{HL})^{\mathrm{m}} \mathrm{H}, \quad(1)
$$

where $\mathrm{H}$ stands for the matrix of the high-refractive index component and $\mathrm{L}$ is the matrix of the low-refractive index component, $m$ and $m o$ are the number of periods for the external and the internal mirror respectively and $\mathrm{L}_{\text {res }}$ is the matrix of the resonator (defect cavity) $\mathrm{L}_{\text {res }}$, which is defined by the following equation $\mathrm{L}_{\text {res }}=\left(2 \pi / \lambda_{\text {res }}\right) \cdot n_{\text {res }} \cdot d_{\text {res }}$ or for non-optimal thickness of the resonator, $d_{\text {res }}=d_{L}$ from the equation $\mathrm{L}_{\text {res }}=\left(2 \pi / \lambda_{\text {res }}\right) \cdot n_{\text {res }} \cdot d_{L}$. The matrices for the interfaces, i.e. IHL, ILH etc. are ignored to simplify the calculations. For calculations, we use various combinations of $n$ in the FP-resonator cavities, with parallel or cross-tuning in the range 1.5-1.7.

The calculation of the matrix and the spectrum of $T$ by means of the Eqn. (1) is performed at the normal incidence of light at $n_{\mathrm{Si}}=3.42$, and the refractive index of incoming $\left(n_{L}\right)$ and outgoing media $(n)$ equal to 1 (related to air). The lattice constant of the reflector is taken as $a=3.4 \mu \mathrm{m}$, the filling factor $f_{\mathrm{Si}}=0.224$, and the width of the resonator cavity $d_{\text {peз }}=2 d_{\mathrm{L}}$. The interaction of localized photonic states leads to the splitting of the optical modes of the system (see Figs.1c and d), which is defined as the difference between two peak positions in the doublet.

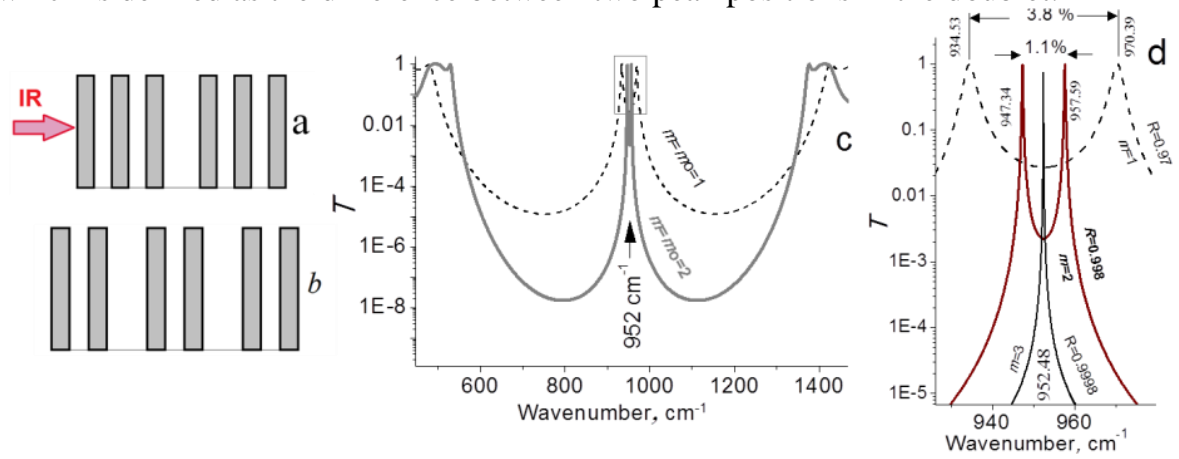

FIGURE 1. (a) Schematic diagram of FP resonator, based on Si-Air with a single defect and a number of periods in the reflectors $m$ $=2$, b) schematic of coupled FP resonators with $m=m o=1$, c) first Stop-Band (SB) in spectrum $T$ with two resonance peaks, and d) magnifying part of doublets from figure (c) and a single peak of FP resonator, similar to one shown in figure (a), but with $m=3$.

Thus, for a resonator with $m=m o=1$ at $\lambda_{0}=10.5 \mu \mathrm{m}$ (i.e. at $v_{0}=952.48 \mathrm{~cm}^{-1}$ ), this splitting corresponds to $\Delta v=$ $35.86 \mathrm{~cm}^{-1}(\Delta \lambda=405 \mathrm{~nm})$, which is $3.8 \%$ with respect to the selected frequency of a single mode in the $1^{\text {st }} \mathrm{SB}\left(v_{0}=\right.$ $\left.952.48 \mathrm{~cm}^{-1}\right)$. As is known [9], the splitting of the doublet depends on the $R$ of the common mirror, which in this case is $R=0.97$, and provides a strong connection between the two oscillators.

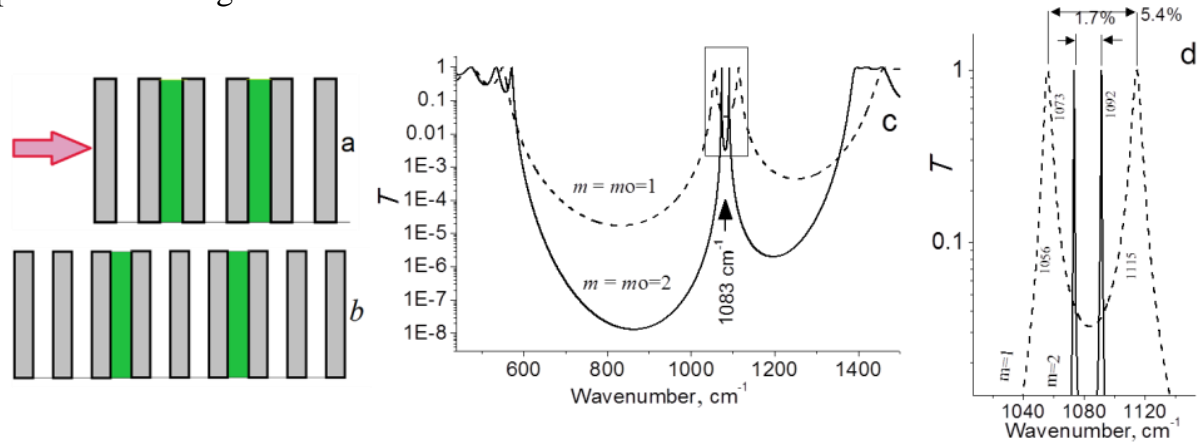

FIGURE 2. Composite coupled resonators transformed from 1D PhC by introducing a filler with $n=1.6$ : a) structure with $m=m o$ $=1, \mathrm{~b})$ structure with $m=m o=2$; c) the transmission spectra of the 1st SB for a doublet of coupled resonators with reflectors having $m=1$ (dashed line) and $m=2$ (solid line); d) a magnifying view of the double resonant peaks from Fig. (c). The numbers beside the peaks indicate the positions of the maxima of the resonant peaks in $\mathrm{cm}^{-1}$. 
In Fig. 1d, a spectrum $T$ of a single-defect cavity resonator was added in order to compare the resonance peak of single defect with doublet peak for the double-cavity FP structures, taking into account an approximately equal number of walls in the reflectors for both cases. It can be seen that the width of the single peak is smaller than for doublet resonances, which is explained by the higher value of $R=0.9998$, due to a larger number of periods of $\mathrm{PhC}$ in the reflectors surrounding a single cavity of the resonator $(m=3)$. The increase in the number of periods in the double-cavity structure up to $m=m o=2$ increases the $R$ of reflectors to 0.998 , weakening the coupling between the oscillators and leading to a decrease in the splitting of peak positions in doublet to $1.1 \%$.

In Ref. [10], it was shown how to transform a $1 \mathrm{D} \mathrm{PhC} \mathrm{into} \mathrm{a} \mathrm{composite} \mathrm{single-cavity} \mathrm{FP} \mathrm{resonator} \mathrm{by} \mathrm{introducing} \mathrm{a}$ liquid crystal into the central $\mathrm{PhC}$ element. We apply this approach to the $1 \mathrm{D} \mathrm{PhC}$ with five periods, as is shown in Fig. $2 \mathrm{a}$, by infiltration the LC filler into the $2^{\text {nd }}$ and $4^{\text {th }}$ periods, as well as for the $1 \mathrm{D} \mathrm{PhC}$ with eight periods, as is shown in Fig. 2b, by infiltration the filler into the $3^{\text {rd }}$ and $6^{\text {th }}$ periods.

The transfer matrix $\mathrm{S}$ for this case is calculated using the Eqn.(1), taking into account the replacement of the value of $n$ by $n_{L C}=1.6$ in $\mathrm{L}_{\text {res } 1}$ and $\mathrm{L}_{\text {res2 }}$. Figure $2 \mathrm{c}$ shows that a doublet, shifted from the center of the $\mathrm{SB}$ (at $v \approx 952 \mathrm{~cm}^{-1}$ ) to the blue region to the value of $v \approx 1083 \mathrm{~cm}^{-1}(\lambda=9.236 \mu \mathrm{m})$ closer to the edge of $\mathrm{SB}$, appeared in the $T$ spectrum in the first stop-band of the composite PhC. The appearance of a doublet not in the center of the SB in both cases is explained by the fact that the optical thicknesses of the defects are not equal to $\lambda_{0} / 2$, where $\lambda_{0}$ was chosen as the center of the first $\mathrm{SB}$ of the reflectors.
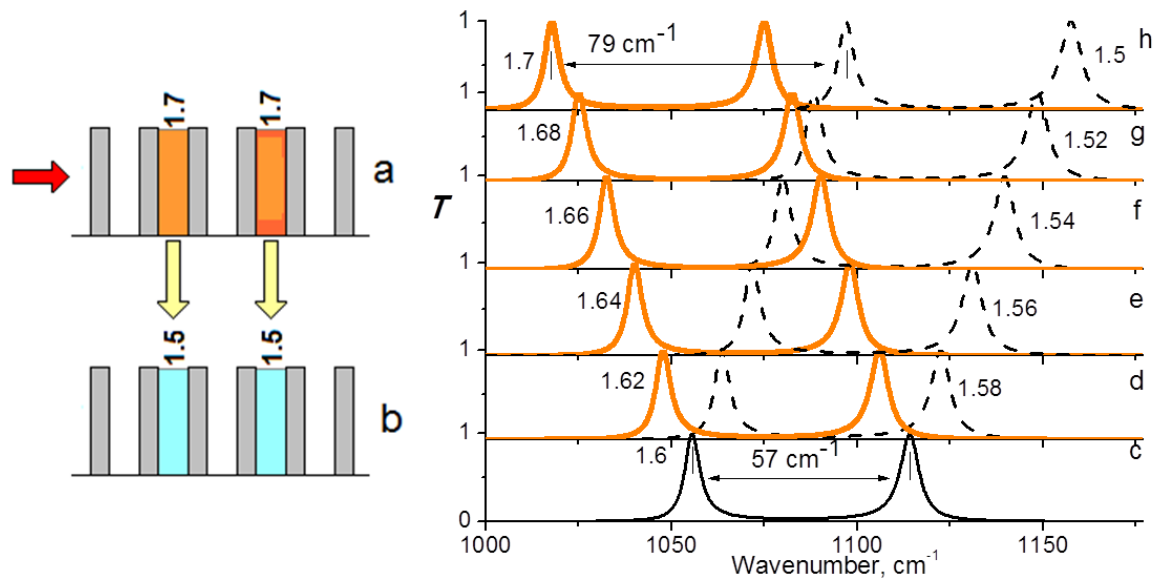

FIGURE 3. (a), (b) Schematic of parallel tuning of the $n_{L C}$ in the range 1.5-1.7 in both cavities of the FP resonator, (c) - (h) doublet peaks in the $T$ spectra for different values of $n_{L C}$ in the cavities of the FP resonators $\left(n_{L C}\right.$ values shown by the numbers beside the peaks).

Taking into account the demonstrated earlier possibility of the electrically controlled change of $n_{L C}$ in a single cavity FP resonator, based on microstructured $\mathrm{Si}$ (see, for example, Ref.[10]), an approach is presented here for tuning of $n_{L C}$ in double-cavities of FP resonator. It is shown that an increase or decrease in $n_{L C}$ leading to different variations in position of doublets of resonance modes.

\section{Different Types of Tuning of the Doublet Modes in LC Resonators}

For tuning the resonance modes, we will change the refractive index $n_{L C}$ in double-cavities of the FP resonator from 1.7 to 1.5 , as schematically shown in Figs. $3 \mathrm{a}$ and $3 \mathrm{~b}$. Considering the possibility of an independent change in $n_{L C}$ in both cavities of the FP resonator, two cases were selected for the study: first - a parallel change in $n_{L C}$ in both cavities (Figs. 3a,b), second - a cross-tuning of $n_{L C}$ in different cavities, that is, changes of $n_{L C}$ occured in opposite directions (Fig. 4a).

The tuning of doublet peaks, depending on the value of $n_{L C}$ varying in parallel in both cavities, is shown for the $1^{\text {st }}$ SB in Figs. 3c-h. Fig. 3c shows only one doublet for $n_{L C}=1.6$, while in all subsequent figures from Fig. 3d to Fig. 3h two spectra are shown: one for $n_{L C}>1.6$ and the second for $n_{L C}<1.6$ to save space. The distance between two peaks in the doublet $\Delta v=57 \mathrm{~cm}^{-1}$ does not change with parallel tuning of the peak positions. As expected, for $n_{L C}=1.7$, the position of the doublet is shifted to the red edge of the spectrum, whilst for $n_{L C}=1.5$ to the blue edge. The total peaks shift reaches $\Delta v=79 \mathrm{~cm}^{-1}$ (Fig. 3h), which results in the relative tuning range per unit refractive index, $\Delta v / \Delta n=4 \mathrm{~cm}^{-1}$. The 
transmission $T$ within the SB region is at the level of no more than 0.001 , while between the doublet peaks it is slightly higher than 0.01 . The transmission value at the maximum of the doublet peaks reaches a value of $T \approx 1$, and the full width of the peaks at half-maximum (FWHM) is $2 \mathrm{~cm}^{-1}$.

In the case of a cross tuning of $n_{L C}$ in two cavities of the FP resonator (see Fig. 4a), the alteration of the doublet position is much smaller: $\Delta v=13 \mathrm{~cm}$, which in relative units is $\Delta v / v=0.011$ (or 1.1\%), Fig. 4b. Below, in Fig. 4c for comparison, the spectrum $T$ is shown at the tuning of $n_{L C}$ in the FP with single cavity, while in Fig. $4 \mathrm{~d}$ two extreme $T$ spectra are presented for the case of parallel tuning of the doublet (from Fig. 3h). In the case of a structure with a single defect, when the $n_{L C}$ is changing from 1.5 to 1.7 , the position of a single peak at $v \approx 1080 \mathrm{~cm}^{-1}$ in the $1^{\text {st }} \mathrm{SB}$ (see Fig. $4 \mathrm{c}$ ) is shifted by the value of $\Delta v=79 \mathrm{~cm}^{-1}(\Delta v / v=7.2 \%)$, which is similar to a shift for one of the doublet peaks (Fig. 3h), with $\Delta v / \Delta n=4 \mathrm{~cm}^{-1}$. The total spectral range occupied by the doublet resonance peaks of the double-cavity structure within SB increased by $57 \mathrm{~cm}^{-1}$ and amounted to $\Delta v=136 \mathrm{~cm}^{-1}$. It should be noted that the width of the $1^{\text {st }} \mathrm{SB}$ is $\Delta v=$ $830 \mathrm{~cm}^{-1}$, that is, when tuning the single-defect structure, less than $10 \%$ of the $R$ bandwidth is used, whilst for the doublet structure this reaches of $16 \%$ of the bandwidth.

Fig. 5a shows a series of spectra demonstrating how the doublet peaks are shifted within $3^{\text {rd }} \mathrm{SB}$ as $n_{L C}$ changes in both cavities of the resonator. The width of the $3^{\text {rd }} \mathrm{SB}$ is $720 \mathrm{~cm}^{-1}$, while the range occupied by the tuned peaks is $\Delta v=$ $390 \mathrm{~cm}^{-1}$, that is, $54 \%$ of SB width. For the initial $n_{L C}=1.6$, the doublet peaks in the cavities of the resonator are in the center of the
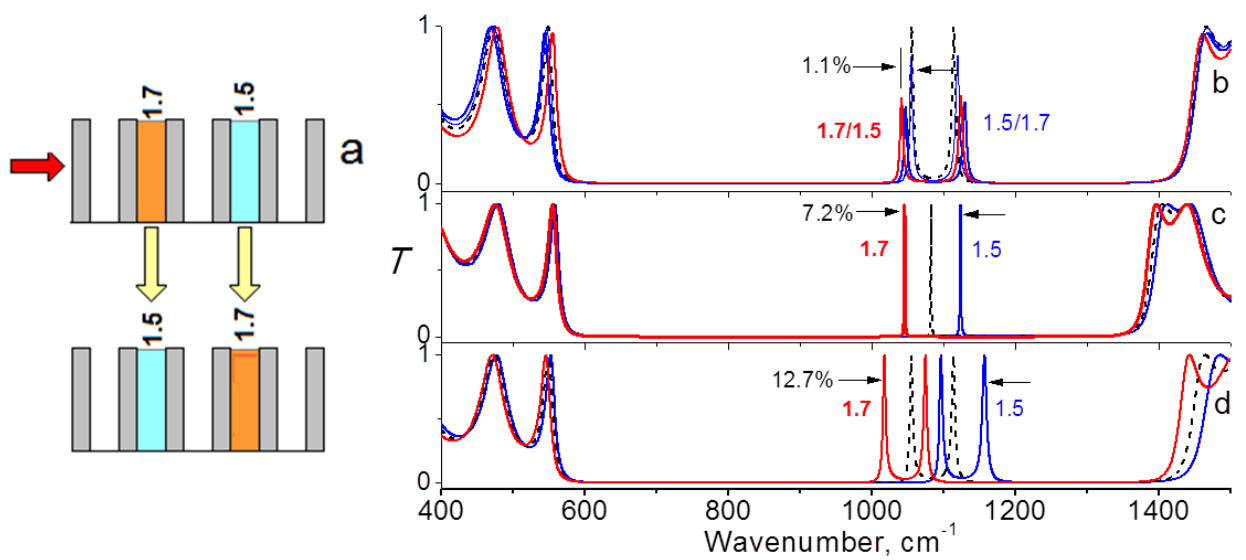

FIGURE 4. a) Schematic of $n_{L C}$ changes in the case of cross-tuning of doublet modes. The relative shift of the resonant peaks in the spectrum of the $1^{\text {st }} \mathrm{SB}$ is shown for the maximum tuning range of $\Delta v_{\max } / v$, given in $\%$ beside the lines: (c) for a single defect, and for two coupled resonators in (b) for cross-tuning and in (d) for parallel tuning of the values of $n_{L C}$ in the cavities of the resonators (numbers beside the peaks).
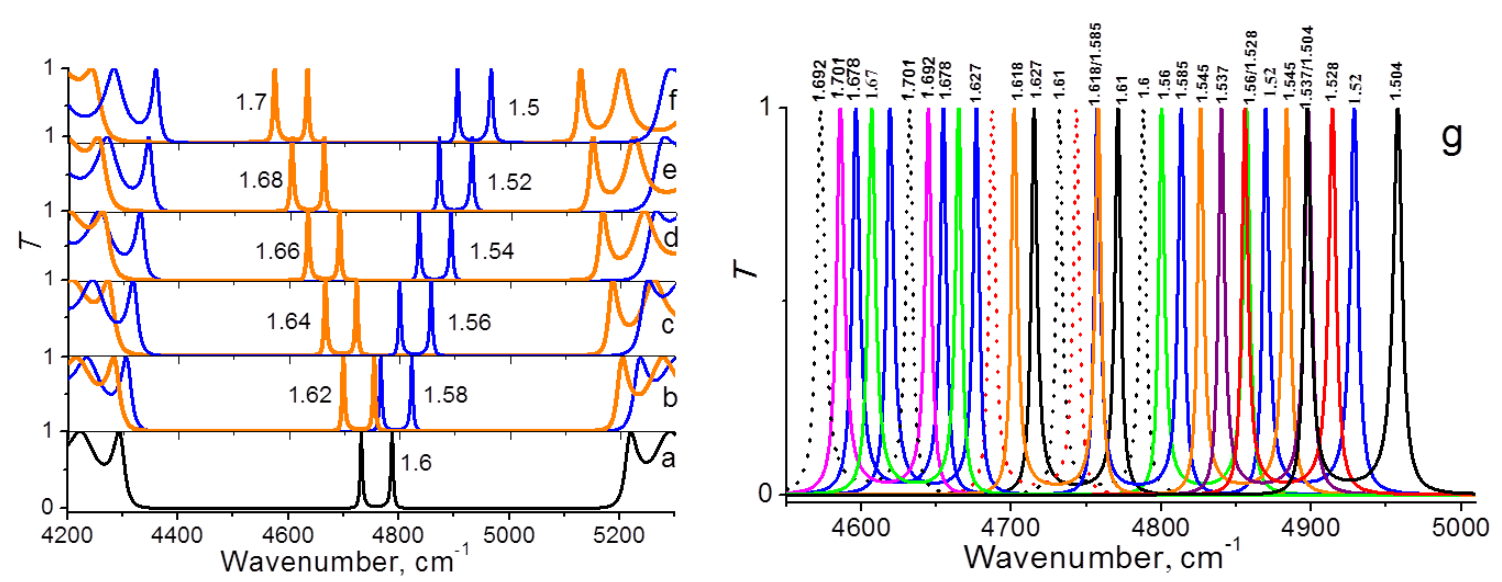

FIGURE 5. a) Tuning of 11 doublet peaks in the $3 \mathrm{rd} \mathrm{SB}$ with a parallel variation of the $n_{L C}$, the numbers beside the peaks are the $n_{L C}$ values in both cavities of the FP resonator; b) demonstration of all doublet pairs obtained within the range of $\sim 390 \mathrm{~cm}^{-1}$ in one graph. Note that three peaks obtained at slightly different $n_{L C}$ are overlapped. 
$\mathrm{SB}$, which enables to reach the maximal possible variation of the number of peaks. The maximum range of the spectrum covered by the tuning of the resonance peaks increased to $\Delta v_{\max }=390 \mathrm{~cm}^{-1}$, that is, almost 3 times of that achieved for the $1^{\text {st }} \mathrm{SB}$. At the same time, the width of each peak increased by no more than twice, namely FWHM $=4 \mathrm{~cm}^{-1}$ for the peak at $v \sim 4800 \mathrm{~cm}^{-1}$. We note that 22 resonance peaks were obtained using eleven possible values of $n_{L C}$ in the range $1.5-1.7$, including the lowest and the highest $n_{L C}$ values (Fig. 5a). However, to obtain the maximum number of non-overlapping peaks, further fine tuning of the $n_{L C}$ within this range is required. For example, the $T$ spectra, obtained with a smaller step of $\Delta n_{L C}$, are summarised in one graph (Fig. 5b) to show all possible doublet peaks (29 peaks in total) that can be obtained within the $3^{\text {rd }} \mathrm{SB}$, taking into account the desired separation of peaks among themselves.

\section{SUMMARY}

In the periodic structure of a one-dimensional photonic crystal the filling of two defined air grooves with a filler of a different refractive index leads to the appearance of two resonant modes, the position of which can be tuned purposefully with changing of the refractive index of the filler. In comparison with air resonators of optical thickness $(\lambda$ /2), the splitting of the doublet increases, which is explained by the increase in the coupling between the resonant modes due to the decrease in $R$ of the internal mirror. Since the main goal when using coupled resonators is the ability to vary the peaks position, structures with a minimum number of periods in a common mirror are considered to provide the maximum possible range of tuning of the position of the resonant peaks. The use of high-order resonances and stop-bands allows us to extend the range of their functionality. Tuning of the doublet peaks in the $1^{\text {st }} \mathrm{SB}$ makes it possible to obtain five pairs of the defect (or resonant) modes, while the use of the $3^{\text {rd }}$ SB provides the increase of their number to 16 pairs. The resonance modes of the obtained structures have high transmission and modulation, good separation between the peaks, and also a considerable range for the choice of the used resonant modes.

\section{ACKNOWLEDGMENT}

We acknowledge financial support from: The Engineering and Physical Sciences Research Council (EPSRC) of the United Kingdom via the EPSRC Grant No. EP/NO35569/1, and the Royal Society International Exchange Grant 2015/R3. The part of this research at the Ioffe Institute was supported by the Russian Federal Agency of Scientific Organizations.

\section{REFERENCES}

1. P.J. Miller, Proc. SPIE, 1235, 466-473 (1990).

2. Y.Yi, P. Bermel, K. Wada, X. Duan, J.D. Joannopoulos, and L.C. Kimerling, Appl. Phys. Lett., 81, $4112-4114$ (2002).

3. Z. Zheng, G. Yang Guowei, H. Li Haifeng, X. Liu, Optics Express, 19, 2158-2164 (2011).

4. G. Shabtay, E. Eidinger, Z. Zalevsky, D. Mendlovic, E Marom, Opt. Express 10, 1534-1541 (2002).

5. O. Aharon, I. Abdulhalim, Opt. Lett., 34, 2114-2116. (2009).

6. D. O’Brien, M.D. Settle, T. Karle, A. Michaeli, M. Salib, T.F. Krauss, Opt. Express, 15, 1228-1233 (2007).

7. K.J Vahala, Nature, 424, 839-846 ( 2003).

8. J. Cos, J. Ferre-Borrull, J. Pallares, L.F. Marsal, Opt. Comm., 282, 1220-1225 (2009).

9. M.A. Kaliteevski, Techn. Phys., 43, 565-568 (1998).

10. V.A. Tolmachev, V.A. Melnikov, A.V. Baldycheva, K. Berwick, and T.S. Perova, PIER, 122, 293-309 (2012). 